MАТНЕМАТІCA, 63 (86), $\mathrm{N}^{\circ}$ 1, 2021, pp. 13-22

\title{
CYCLES OF REDUCED IDEALS AND CLASS NUMBER OF PURE CUBIC NUMBER FIELD
}

\author{
ABDELMALEK AZIZI, JAMAL BENAMARA, MOULAY CHRIF ISMAILI, \\ and MOHAMMED TALBI
}

\begin{abstract}
For some pure cubic field $K$, we compute the class number of $K$ based on the notion of reduced ideals and the notion of the minimum of an ideal. MSC 2010. Primary 11R16, 11R29; Secondary $11 T 71$.

Key words. Cubic field, minimum of ideal, reduced ideal, cycle of reduced ideal.
\end{abstract}

\section{REFERENCES}

[1] H. Appelgate and H. Onishi, Periodic expansions of modules and its relation to units, J. Number Theory, 15 (1982), 283-294.

[2] J. Buchmann, On the computation of units and class numbers by a generalization of Lagrange's algorithm, J. Number Theory, 26 (1987), 8-30.

[3] H. Cohen, A course in computational algebraic number theory, 3rd ed., Springer-Verlag, 1996.

[4] G.T. Jacobs, Reduced ideals in pure cubic fields, arXiv:1905.00242v3.

[5] R.A. Mollin, Quadratics, CRC Press, Boca Raton, 1996.

[6] J. Neukirch, Algebraic number theory, Springer-Verlag, New York, 1999.

[7] J.J. Payan, Sur le groupe des classes d'un corps quadratique, Cours de l'institut Fourier, 7 (1972), 2-30.

Received June 20, 2019

Accepted May 22, 2020

\author{
Mohammed First University \\ Faculty of Sciences \\ Department of Mathematics \\ 60000, Oujda, Morocco \\ E-mail: abdelmalekazizi@yahoo.fr \\ https://orcid.org/0000-0002-0634-1995 \\ E-mail: benamarajamal@hotmail.fr \\ https : //orcid.org/0000-0003-0303-0355 \\ E-mail: mcismaili@yahoo.fr \\ Regional center of Education and Training \\ 60000, Oujda, Morocco \\ E-mail: talbimm@yahoo.fr \\ https://orcid.org/0000-0001-5430-3144
}

DOI: 10.24193/mathcluj.2021.1.02 